\title{
The harmonic index for unicyclic and bicyclic graphs with given matching number
}

\author{
Lingping Zhong
}




\title{
THE HARMONIC INDEX FOR UNICYCLIC AND BICYCLIC GRAPHS WITH GIVEN MATCHING NUMBER
}

\author{
LINGPING ZHONG
}

Received 24 October, 2013

\begin{abstract}
The harmonic index of a graph $G$ is defined as the sum of the weights $\frac{2}{d(u)+d(v)}$ of all edges $u v$ of $G$, where $d(u)$ denotes the degree of a vertex $u$ in $G$. In this paper, we present the minimum harmonic indices for unicyclic and bicyclic graphs with $n$ vertices and matching number $m\left(2 \leq m \leq\left\lfloor\frac{n}{2}\right\rfloor\right)$, respectively. The corresponding extremal graphs are also characterized.
\end{abstract}

2000 Mathematics Subject Classification: 05C07; 05C70; 05C35; 92E10

Keywords: harmonic index, unicyclic graph, bicyclic graph, matching number

\section{INTRODUCTION}

Let $G$ be a simple graph with vertex set $V(G)$ and edge set $E(G)$. The Randić index $R(G)$, proposed by Randić [20] in 1975, is defined as

$$
R(G)=\sum_{u v \in E(G)} \frac{1}{\sqrt{d(u) d(v)}}
$$

where $d(u)$ denotes the degree of a vertex $u$ of $G$. The Randic index is one of the most successful molecular descriptors in structure-property and structure-activity relationship studies. Mathematical properties of this descriptor have been studied extensively (see $[9,10,14,15,19]$ and the references cited therein).

In this paper, we consider a closely related variant of the Randić index, named the harmonic index. For a graph $G$, the harmonic index $H(G)$ is defined as

$$
H(G)=\sum_{u v \in E(G)} \frac{2}{d(u)+d(v)} .
$$

This index first appeared in [6], and it can also be viewed as a particular case of the general sum-connectivity index proposed by Zhou and Trinajstić in [32].

This work was supported by the Fundamental Research Funds for the Central Universities (No. NS2015078). 
Favaron, Mahéo and Saclé [7] considered the relation between the harmonic index and the eigenvalues of graphs. Zhong [28, 29], Zhong and Xu [30] determined the minimum and maximum harmonic indices for simple connected graphs, trees, unicyclic and bicyclic graphs, and characterized the corresponding extremal graphs. $\mathrm{Wu}$, Tang and Deng [23] found the minimum harmonic index for graphs (triangle-free graphs, respectively) with minimum degree at least 2, and characterized the corresponding extremal graphs. Deng, Balachandran, Ayyaswamy and Venkatakrishnan [2] considered the relation between the harmonic index and the chromatic number of a graph by using the effect of removal of a minimum degree vertex on the harmonic index. Liu [17] proposed a conjecture concerning the relation between the harmonic index and the diameter of a connected graph, and showed that the conjecture is true for trees. Ilić [12], Xu [25], Zhong and Xu [31] established some relationships between the harmonic index and several other topological indices. The chemical applicability of the harmonic index was also recently investigated $[8,11]$. See $[3,18,24,26]$ for more information of this index.

In this paper, we determine the minimum harmonic indices for unicyclic and bicyclic graphs with $n$ vertices and matching number $m\left(2 \leq m \leq\left\lfloor\frac{n}{2}\right\rfloor\right)$, respectively. The corresponding extremal graphs are also characterized. The related problems have been well-studied for several other topological indices, such as the Randić index [16,33], the modified Randić index [13] and the sum-connectivity index [4,5,21,22].

\section{PRELIMinaries}

Let $G$ be a graph. For any vertex $v \in V(G)$, we use $N_{G}(v)$ (or $N(v)$ if there is no ambiguity) to denote the set of neighbors of $v$ in $G$. A pendent vertex is a vertex of degree 1. For two distinct vertices $u$ and $v$ of $G$, the distance $d(u, v)$ between $u$ and $v$ is the number of edges in a shortest path joining $u$ and $v$ in $G$. A unicyclic graph is a connected graph with $n$ vertices and $n$ edges, and a bicyclic graph is a connected graph with $n$ vertices and $n+1$ edges. We use $C_{n}$ to denote the cycle on $n$ vertices.

A matching $M$ in a graph $G$ is a subset of $E(G)$ such that no two edges in $M$ share a common vertex. A matching $M$ in $G$ is said to be maximum, if for any other matching $M^{\prime}$ in $G,\left|M^{\prime}\right| \leq|M|$. The matching number of $G$ is the number of edges in a maximum matching of $G$. If $M$ is a matching in $G$ and the vertex $v \in V(G)$ is incident with an edge of $M$, then $v$ is said to be $M$-saturated, and if every vertex in $G$ is $M$-saturated, then $M$ is a perfect matching.

For any vertex $v \in V(G)$, we use $G-v$ to denote the graph resulting from $G$ by deleting the vertex $v$ and its incident edges. We define $G-u v$ to be the graph obtained from $G$ by deleting the edge $u v \in E(G)$, and $G+u v$ to be the graph obtained from $G$ by adding an edge $u v$ between two non-adjacent vertices $u$ and $v$ of $G$.

We now establish some lemmas which will be used frequently in later proofs. 
Lemma 1. Let $G$ be a connected graph on $n \geq 4$ vertices with a pendent vertex $u$. Let $v$ be the unique neighbor of $u$ with $d(v)=s$, and let $w$ be a neighbor of $v$ different from $u$ with $d(w)=t$.

( $i$ ) If $s=2$ and $w$ is adjacent to at most one pendent vertex in $G$, then

$$
H(G) \geq H(G-u-v)+\frac{2(t-1)}{t+2}-\frac{2(t-3)}{t+1}-\frac{2}{t}+\frac{2}{3}
$$

with equality if and only if one neighbor of $w$ has degree 1 and the other neighbors of $w$ have degree 2.

(ii) If $v$ is adjacent to at most $k$ pendent vertices in $G$, then

$$
H(G) \geq H(G-u)+\frac{2(s-k)}{s+2}+\frac{2(2 k-s)}{s+1}-\frac{2(k-1)}{s}
$$

with equality if and only if $k$ neighbors of $v$ have degree 1 and the other neighbors of $v$ have degree 2.

Proof. (i) Let $N(w)=\left\{w_{0}=v, w_{1}, \ldots, w_{t-1}\right\}$. Since $w$ is adjacent to at most one pendent vertex in $G$, we may assume that $d\left(w_{1}\right) \geq 1$, and $d\left(w_{i}\right) \geq 2$ for each $2 \leq i \leq t-1$ (if $t \geq 3$ ). Note that $\frac{2}{t+x}-\frac{2}{t-1+x}$ is increasing for $x \geq 1$, we have

$$
\begin{aligned}
H(G) & =H(G-u-v)+\sum_{i=1}^{t-1}\left(\frac{2}{t+d\left(w_{i}\right)}-\frac{2}{t-1+d\left(w_{i}\right)}\right)+\frac{2}{t+2}+\frac{2}{3} \\
& \geq H(G-u-v)+\left(\frac{2}{t+1}-\frac{2}{t}\right)+(t-2)\left(\frac{2}{t+2}-\frac{2}{t+1}\right)+\frac{2}{t+2}+\frac{2}{3} \\
& =H(G-u-v)+\frac{2(t-1)}{t+2}-\frac{2(t-3)}{t+1}-\frac{2}{t}+\frac{2}{3}
\end{aligned}
$$

with equality if and only if $d\left(w_{1}\right)=1$ and $d\left(w_{i}\right)=2$ for each $2 \leq i \leq t-1$ (if $t \geq 3$ ). This proves (i).

(ii) Let $r(1 \leq r \leq k)$ be the number of pendent neighbors of $v$ in $G$, and let $N(v)=$ $\left\{v_{0}=u, v_{1}, \ldots, v_{s-1}\right\}$. Without loss of generality, we may assume that $d\left(v_{i}\right)=1$ for each $1 \leq i \leq r-1$ (if $r \geq 2$ ), and $d\left(v_{i}\right) \geq 2$ for each $r \leq i \leq s-1$ (if $s \geq r+1$ ). Note that $\frac{2}{s+x}-\frac{2}{s-1+x}$ is increasing for $x \geq 1$ and $\frac{4}{s+1}-\frac{\overline{2}}{s+2}-\frac{2}{s}<0$, we have

$$
\begin{aligned}
H(G)= & H(G-u)+(r-1)\left(\frac{2}{s+1}-\frac{2}{s}\right) \\
& +\sum_{i=r}^{s-1}\left(\frac{2}{s+d\left(v_{i}\right)}-\frac{2}{s-1+d\left(v_{i}\right)}\right)+\frac{2}{s+1} \\
\geq & H(G-u)+(r-1)\left(\frac{2}{s+1}-\frac{2}{s}\right)+(s-r)\left(\frac{2}{s+2}-\frac{2}{s+1}\right)+\frac{2}{s+1} \\
= & H(G-u)+r\left(\frac{4}{s+1}-\frac{2}{s+2}-\frac{2}{s}\right)+\frac{2 s}{s+2}-\frac{2 s}{s+1}+\frac{2}{s}
\end{aligned}
$$




$$
\begin{aligned}
& \geq H(G-u)+k\left(\frac{4}{s+1}-\frac{2}{s+2}-\frac{2}{s}\right)+\frac{2 s}{s+2}-\frac{2 s}{s+1}+\frac{2}{s} \\
& =H(G-u)+\frac{2(s-k)}{s+2}+\frac{2(2 k-s)}{s+1}-\frac{2(k-1)}{s}
\end{aligned}
$$

with equalities if and only if $r=k$ and $d\left(v_{i}\right)=2$ for each $k \leq i \leq s-1$ (if $s \geq k+1$ ). This completes the proof of the lemma.

Lemma 2. (i) The function $\frac{2(x-1)}{x+2}-\frac{2(x-3)}{x+1}-\frac{2}{x}$ is decreasing for $x \geq 2$.

(ii) For $k \geq 1$, the function $\frac{2(x-k)}{x+2}+\frac{2(2 k-x)}{x+1}-\frac{2(k-1)}{x}$ is decreasing for $x \geq$ $k+1$.

Proof. (i) Let $f(x)=\frac{2(x-1)}{x+2}-\frac{2(x-3)}{x+1}-\frac{2}{x}=\frac{8}{x+1}-\frac{6}{x+2}-\frac{2}{x}$. For $x \geq 2$, we have

$$
\begin{aligned}
f^{\prime}(x) & =-\frac{8}{(x+1)^{2}}+\frac{6}{(x+2)^{2}}+\frac{2}{x^{2}}=\frac{-8 x^{3}+24 x+8}{x^{2}(x+1)^{2}(x+2)^{2}} \\
& =\frac{-8 x\left(x^{2}-4\right)-8(x-1)}{x^{2}(x+1)^{2}(x+2)^{2}}<0,
\end{aligned}
$$

and hence (i) holds.

(ii) Let $g(x)=\frac{2(x-k)}{x+2}+\frac{2(2 k-x)}{x+1}-\frac{2(k-1)}{x}$ and $g_{1}(x)=\frac{2(k-1)}{x}+\frac{2(x-1-k)}{x+1}$. Then $g(x)=g_{1}(x+1)-g_{1}(x)$. For $x \geq k+1 \geq 2$, we have

$$
\begin{aligned}
g_{1}^{\prime \prime}(x) & =\frac{4(k-1)}{x^{3}}-\frac{4(k+2)}{(x+1)^{3}}=\frac{-12 x^{3}+12(k-1) x^{2}+12(k-1) x+4(k-1)}{x^{3}(x+1)^{3}} \\
& =\frac{-12 x^{2}(x-k)-12 x(x-k)-4(3 x-k+1)}{x^{3}(x+1)^{3}}<0,
\end{aligned}
$$

and $g^{\prime}(x)=g_{1}^{\prime}(x+1)-g_{1}^{\prime}(x)<0$. So the assertion of the lemma holds.

Lemma 3. Let $G$ be a connected graph, and let $u$ be a vertex of degree 2 in $G$ with two neighbors $v$ and $w$ such that $d(v) \geq 2$ and $v w \notin E(G)$. Let $G^{\prime}=G-u w+v w$, then $H(G)>H\left(G^{\prime}\right)$.

Proof. Let $d(v)=p \geq 2$ and let $N(v)=\left\{v_{0}=u, v_{1}, \ldots, v_{p-1}\right\}$. Then

$$
\begin{aligned}
& H(G)-H\left(G^{\prime}\right) \\
& =\left(\sum_{i=1}^{p-1} \frac{2}{p+d\left(v_{i}\right)}+\frac{2}{2+d(w)}\right)-\left(\sum_{i=1}^{p-1} \frac{2}{p+1+d\left(v_{i}\right)}+\frac{2}{p+1+d(w)}\right) \\
& =\sum_{i=1}^{p-1}\left(\frac{2}{p+d\left(v_{i}\right)}-\frac{2}{p+1+d\left(v_{i}\right)}\right)+\left(\frac{2}{2+d(w)}-\frac{2}{p+1+d(w)}\right)>0 .
\end{aligned}
$$

This proves the lemma. 


\section{Minimum HARMONIC INDEX FOR UNICYCLIC GRAPHS WITH GIVEN MATCHING NUMBER}

Let $\mathscr{U}_{n}$ be the set of unicyclic graphs with $n \geq 3$ vertices, and let $\mathscr{U}_{n, m}$ be the set of unicyclic graphs with $n$ vertices and matching number $m$, where $2 \leq m \leq\left\lfloor\frac{n}{2}\right\rfloor$. In this section, we determine the minimum harmonic index for graphs in $\mathscr{U}_{n, m}$, and characterize the corresponding extremal graphs.

For a unicyclic graph $G$ with the cycle $C_{p}$, the forest obtained from $G$ by deleting the edges in $C_{p}$ consists of $p$ vertex-disjoint trees, each containing a vertex of $C_{p}$, which is called the root of this tree in $G$. These trees are called the branches of $G$. Chang and Tian [1] showed the following lemma.

Lemma 4. Let $G \in \mathscr{U}_{2 m, m}(m \geq 3)$, and let $T$ be a branch of $G$ with root $r$. If $u \in V(T)$ is a pendent vertex which is furthest from the root $r$ with $d(u, r) \geq 2$, then $u$ is adjacent to a vertex of degree 2 .

The second lemma was proved by Yu and Tian [27].

Lemma 5. Let $G \in \mathscr{U}_{n, m}(n>2 m)$ and $G \not C_{n}$. Then there exists a maximum matching $M$ and a pendant vertex $u$ in $G$ such that $u$ is not $M$-saturated.

Zhong [29] proved the following result.

Lemma 6. Let $G \in \mathscr{U}_{n}$ with $n \geq 3$. Then $H(G) \leq \frac{n}{2}$ with equality if and only if $G \cong C_{n}$.

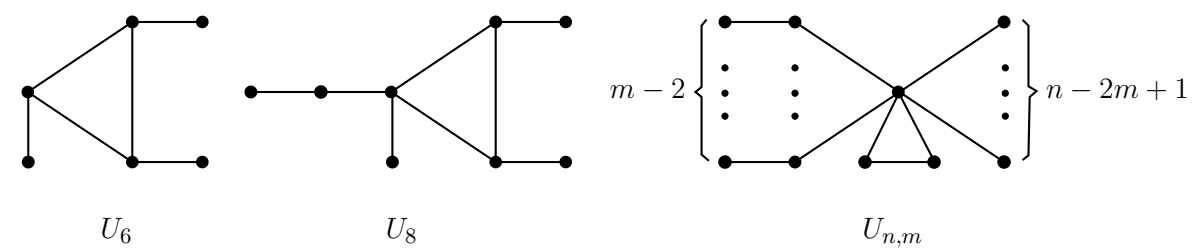

FIGURE 1. The graphs $U_{6}, U_{8}$ and $U_{n, m}$.

Let $U_{6}$ be the unicyclic graph on 6 vertices obtained by attaching a pendent vertex to every vertex of a triangle, and let $U_{8}$ be the unicyclic graph on 8 vertices obtained by attaching a path on two vertices to one vertex of degree 3 of $U_{6}$. For $2 \leq m \leq\left\lfloor\frac{n}{2}\right\rfloor$, we use $U_{n, m}$ to denote the unicyclic graph on $n$ vertices obtained by attaching $n-$ $2 m+1$ pendent vertices and $m-2$ paths on two vertices to one vertex of a triangle. See Figure 1 for an illustration.

Theorem 1. Let $G \in \mathscr{U}_{2 m, m} \backslash\left\{U_{6}, U_{8}\right\}$, where $m \geq 2$. Then

$$
H(G) \geq \frac{2 m}{m+3}+\frac{2}{m+2}+\frac{2(m-2)}{3}+\frac{1}{2}
$$

with equality if and only if $G \cong U_{2 m, m}$. 
Proof. We prove the theorem by induction on $m$. If $m=2$, then either $G \cong C_{4}$ or $G \cong U_{4,2}$. Since $H\left(C_{4}\right)=2>\frac{9}{5}=H\left(U_{4,2}\right)$, we see that the assertion of the theorem holds. So we may assume that $m \geq 3$ and the result holds for graphs in $\mathscr{U}_{2(m-1), m-1} \backslash\left\{U_{6}, U_{8}\right\}$. By Lemma 6 , since $C_{2 m}$ is the unique unicyclic graph on $2 m$ vertices with the maximum harmonic index, we may further assume that $G \not$ $C_{2 m}$. Let $M$ be a maximum matching in $G$, then $|M|=m$. By Lemma 4, we need only consider the following two cases.

Case 1. There exists a pendent vertex $u$ in $G$ which is adjacent to a vertex $v$ of degree 2 .

Let $w$ be the neighbor of $v$ different from $u$ with $d(w)=t \geq 2$, and let $G^{\prime}=$ $G-u-v$. Then $u v \in M$ and $G^{\prime} \in \mathscr{U}_{2(m-1), m-1}$. Since $M$ contains exactly one edge incident with $w$ and there are $m$ edges of $G$ outside $M$, we have $t \leq m+1$. Note that $w$ is adjacent to at most one pendent vertex in $G$.

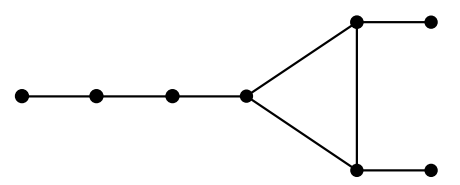

$W_{1}$

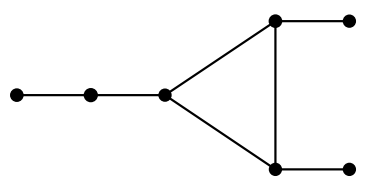

$W_{2}$

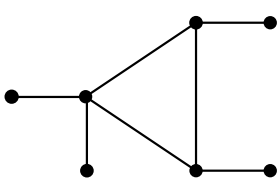

$W_{3}$

FIGURE 2. The graphs $W_{1}, W_{2}$ and $W_{3}$.

If $G^{\prime} \cong U_{6}$, then we have $G \cong W_{1}$ (since we assume $G \not U_{8}$ ), see Figure 2. Since $H\left(W_{1}\right)=\frac{107}{30}>\frac{139}{42}=H\left(U_{8,4}\right)$, we know that the result holds.

If $G^{\prime} \cong U_{8}$, then $t \leq 5$. By Lemma 1(i) and Lemma 2(i), we have

$$
\begin{aligned}
H(G) & \geq H\left(U_{8}\right)+\frac{2(t-1)}{t+2}-\frac{2(t-3)}{t+1}-\frac{2}{t}+\frac{2}{3} \\
& \geq \frac{347}{105}+\frac{2 \cdot(5-1)}{5+2}-\frac{2 \cdot(5-3)}{5+1}-\frac{2}{5}+\frac{2}{3}=\frac{85}{21}>\frac{113}{28}=H\left(U_{10,5}\right),
\end{aligned}
$$

and hence the assertion of the theorem holds.

Now suppose that $G^{\prime} \not U_{6}, U_{8}$. Then by Lemma 1(i), Lemma 2(i) and the induction hypothesis, we conclude that

$$
\begin{aligned}
H(G) \geq & H\left(G^{\prime}\right)+\frac{2(t-1)}{t+2}-\frac{2(t-3)}{t+1}-\frac{2}{t}+\frac{2}{3} \\
\geq & \left(\frac{2(m-1)}{(m-1)+3}+\frac{2}{(m-1)+2}+\frac{2[(m-1)-2]}{3}+\frac{1}{2}\right) \\
& +\frac{2[(m+1)-1]}{(m+1)+2}-\frac{2[(m+1)-3]}{(m+1)+1}-\frac{2}{m+1}+\frac{2}{3}
\end{aligned}
$$




$$
=\frac{2 m}{m+3}+\frac{2}{m+2}+\frac{2(m-2)}{3}+\frac{1}{2}
$$

with equalities if and only if $G^{\prime} \cong U_{2(m-1), m-1}$ and $t=m+1$, i.e., $G \cong U_{2 m, m}$. This proves Case 1.

Case 2. $G$ is a unicyclic graph with maximum degree 3 obtained by attaching $2 m-p$ pendent vertices to some vertices of a cycle $C_{p}(m \leq p \leq 2 m-1)$.

If $m=3$, then $G$ is either the unicyclic graph obtained by attaching a pendent vertex to one vertex of $C_{5}$ or the unicyclic graph obtained by attaching a pendent vertex to two adjacent vertices of $C_{4}$ (since we assume $G \not U_{6}$ ). Then we have $H(G) \geq \frac{79}{30}>\frac{77}{30}=H\left(U_{6,3}\right)$, and the theorem holds. So we may assume that $m \geq 4$. We consider two subcases according to the value of $p$.

Subcase 2.1. $p=m$.

Then every vertex of $C_{p}$ is attached by a pendent vertex and $H(G)=\frac{5 m}{6}$. Let $f(x)=\frac{5 x}{6}-\left(\frac{2 x}{x+3}+\frac{2}{x+2}+\frac{2(x-2)}{3}+\frac{1}{2}\right)=\frac{x}{6}+\frac{6}{x+3}-\frac{2}{x+2}-\frac{7}{6}$. For $x \geq 4$, we have

$$
f^{\prime}(x)=\frac{1}{6}-\frac{6}{(x+3)^{2}}+\frac{2}{(x+2)^{2}} \geq \frac{1}{6}-\frac{6}{(4+3)^{2}}+\frac{2}{(x+2)^{2}}>0 .
$$

This implies that $f(x)$ is increasing for $x \geq 4$, and thus $f(m) \geq f(4)=\frac{1}{42}>0$, i.e., $H(G)>H\left(U_{2 m, m}\right)$.

Subcase 2.2. $m+1 \leq p \leq 2 m-1$.

In this subcase, there exists at least one edge, say $x y$, on $C_{p}$ such that $x y \in M$. Then $d(x)=d(y)=2$; for otherwise, the pendent vertex adjacent to $x$ or $y$ can not be $M$-saturated. Let $z$ be the neighbor of $x$ different from $y$ in $G$, and let $G^{\prime \prime}=G-x z+$ $y z$. Then $G^{\prime \prime} \in \mathscr{U}_{2 m, m} \backslash\left\{U_{8}\right\}$. By Lemma 3, we have $H(G)>H\left(G^{\prime \prime}\right)$. Comparing with the graph $G$, we see that the length of the unique cycle in $G^{\prime \prime}$ decreases by 1 . Repeating this operation from $G$ to $G^{\prime \prime}$, we eventually obtain the unicyclic graph described in Subcase 2.1 and the result holds. This finishes the proof of the theorem.

Since $H\left(U_{6,3}\right)=\frac{77}{30}>\frac{5}{2}=H\left(U_{6}\right)$ and $H\left(U_{8,4}\right)=\frac{139}{42}>\frac{347}{105}=H\left(U_{8}\right)$, by Theorem 1 , we immediately obtain the following two results.

Corollary 1. Let $G \in \mathscr{U}_{6,3}$, then $H(G) \geq \frac{5}{2}$ with equality if and only if $G \cong U_{6}$.

Corollary 2. Let $G \in \mathscr{U}_{8,4}$, then $H(G) \geq \frac{347}{105}$ with equality if and only if $G \cong U_{8}$.

We now prove the main result of this section.

Theorem 2. Let $G \in \mathscr{U}_{n, m} \backslash\left\{U_{6}, U_{8}\right\}$, where $2 \leq m \leq\left\lfloor\frac{n}{2}\right\rfloor$. Then

$$
H(G) \geq \frac{2 m}{n-m+3}+\frac{2(n-2 m+1)}{n-m+2}+\frac{2(m-2)}{3}+\frac{1}{2}
$$

with equality if and only if $G \cong U_{n, m}$. 
Proof. We prove Theorem 2 by induction on $n$. If $n=2 m$, then by Theorem 1 , the assertion of the theorem holds. So we may assume that $n>2 m$ and the result holds for graphs in $\mathscr{U}_{n-1, m} \backslash\left\{U_{6}, U_{8}\right\}$. By Lemma 6 , since $C_{n}$ is the unique unicyclic graph on $n$ vertices with the maximum harmonic index, we may also assume that $G \not C_{n}$. Then by Lemma 5, there exists a maximum matching $M$ and a pendant vertex $u$ in $G$ such that $u$ is not $M$-saturated. Let $v$ be the unique neighbor of $u$ with $d(v)=s \geq 2$, and let $G^{\prime}=G-u$. Then $G^{\prime} \in \mathscr{U}_{n-1, m}$. Since $M$ contains exactly one edge incident with $v$ and there are $n-m$ edges of $G$ outside $M$, we have $s \leq n-m+1$. Let $r$ be the number of pendant neighbors of $v$ in $G$, where $1 \leq r \leq s-1$. Note that at least $r-1$ pendant neighbors of $v$ are not $M$-saturated, and there are $n-2 m$ vertices are not $M$-saturated in $G$. Then $r \leq n-2 m+1$.

If $G^{\prime} \cong U_{6}$, then $n=7, m=3$ and either $G \cong W_{2}$ or $G \cong W_{3}$ (see Figure 2). Since $H\left(W_{2}\right)=\frac{46}{15}>H\left(W_{3}\right)=\frac{284}{105}>\frac{113}{42}=H\left(U_{7,3}\right)$, we see that the result holds.

If $G^{\prime} \cong U_{8}$, then $n=9, m=4$ and $s \leq 5$. By Lemma 1(ii) (with $k=n-2 m+1=$ 2) and Lemma 2(ii), we have

$$
\begin{aligned}
H(G) & \geq H\left(U_{8}\right)+\frac{2(s-2)}{s+2}+\frac{2(4-s)}{s+1}-\frac{2}{s} \\
& \geq \frac{347}{105}+\frac{2 \cdot(5-2)}{5+2}+\frac{2 \cdot(4-5)}{5+1}-\frac{2}{5}=\frac{24}{7}>\frac{143}{42}=H\left(U_{9,4}\right),
\end{aligned}
$$

and thus the assertion of the theorem holds.

Therefore we may assume that $G^{\prime} \not U_{6}, U_{8}$. Then by Lemma 1(ii) (with $k=$ $n-2 m+1$ ), Lemma 2(ii) and the induction hypothesis, we conclude that

$$
\begin{aligned}
H(G) \geq & H\left(G^{\prime}\right)+\frac{2[s-(n-2 m+1)]}{s+2}+\frac{2[2(n-2 m+1)-s]}{s+1} \\
& -\frac{2[(n-2 m+1)-1]}{s} \\
\geq & \left(\frac{2 m}{(n-1)-m+3}+\frac{2[(n-1)-2 m+1]}{(n-1)-m+2}+\frac{2(m-2)}{3}+\frac{1}{2}\right) \\
& +\frac{2[(n-m+1)-(n-2 m+1)]}{(n-m+1)+2}+\frac{2[2(n-2 m+1)-(n-m+1)]}{(n-m+1)+1} \\
& -\frac{2[(n-2 m+1)-1]}{n-m+1} \\
= & \frac{2 m}{n-m+3}+\frac{2(n-2 m+1)}{n-m+2}+\frac{2(m-2)}{3}+\frac{1}{2}
\end{aligned}
$$

with equalities if and only if $G^{\prime} \cong U_{n-1, m}, s=n-m+1$ and $r=n-2 m+1$, i.e., $G \cong U_{n, m}$. This completes the proof of the theorem.

By applying Theorem 2, we can also obtain the minimum harmonic index for graphs in $\mathscr{U}_{n}(n \geq 4)$. This is one of the main results in [29]. 
Corollary 3. Let $G \in \mathscr{U}_{n}$ with $n \geq 4$. Then

$$
H(G) \geq \frac{4}{n+1}+\frac{2(n-3)}{n}+\frac{1}{2}
$$

with equality if and only if $G \cong U_{n, 2}$.

Proof. Let $M$ be a maximum matching in $G$, then $2 \leq|M|=m \leq\left\lfloor\frac{n}{2}\right\rfloor$ (since $n \geq 4)$. If $m=2$, then by Theorem 2 , we have

$$
\begin{aligned}
H(G) & \geq \frac{2 \cdot 2}{n-2+3}+\frac{2(n-2 \cdot 2+1)}{n-2+2}+\frac{2 \cdot(2-2)}{3}+\frac{1}{2} \\
& =\frac{4}{n+1}+\frac{2(n-3)}{n}+\frac{1}{2}
\end{aligned}
$$

with equality if and only if $G \cong U_{n, 2}$. So we may assume that $m \geq 3$.

If $G \cong U_{6}$, then $H(G)=\frac{5}{2}>\frac{29}{14}=H\left(U_{6,2}\right)$, we see that the result holds. If $G \cong U_{8}$, then $H(G)=\frac{347}{105}>\frac{79}{36}=H\left(U_{8,2}\right)$, and the result also holds. Now suppose that $G \not U_{6}, U_{8}$. Then by Theorem 2 and Lemma 3, we see that $H(G) \geq H\left(U_{n, m}\right)>$ $H\left(U_{n, m-1}\right)>\cdots>H\left(U_{n, 2}\right)$. So the assertion of the corollary holds.

\section{Minimum HARMONIC INDEX FOR BICYCLIC GRAPHS WITH GIVEN MATCHING NUMBER}

Let $\mathscr{B}_{n}$ be the set of bicyclic graphs with $n \geq 4$ vertices, and let $\mathscr{B}_{n, m}$ be the set of bicyclic graphs with $n$ vertices and matching number $m$, where $2 \leq m \leq\left\lfloor\frac{n}{2}\right\rfloor$. In this section, we present the minimum harmonic index for graphs in $\mathscr{B}_{n, m}$, and characterize the corresponding extremal graphs.

We denote by $\tilde{\mathscr{B}}_{n}$ the set of bicyclic graphs with $n \geq 4$ vertices containing no pendent vertices. Let $\mathscr{B}_{n}^{1}$ be the set of bicyclic graphs on $n \geq 6$ vertices obtained by connecting two vertex-disjoint cycles by a new edge, and let $\mathscr{B}_{n}^{2}$ be the set of bicyclic graphs on $n \geq 7$ vertices obtained by connecting two vertex-disjoint cycles by a path of length at least two. Let $\mathscr{B}_{n}^{3}$ be the set of bicyclic graphs on $n \geq 5$ vertices obtained by identifying a vertex of a cycle and a vertex of the other cycle. Let $\mathscr{B}_{n}^{4}$ be the set of bicyclic graphs on $n \geq 4$ obtained from $C_{n}$ by adding a new edge, and let $\mathscr{B}_{n}^{5}$ be the set of bicyclic graphs on $n \geq 5$ obtained by connecting two non-adjacent vertices by a path of length at least two. Clearly, $\tilde{\mathscr{B}}_{n}=\bigcup_{i=1}^{5} \mathscr{B}_{n}^{i}$.

For $i=4,5$, we use $B_{i}$ to denote the unique bicyclic graph on $i$ vertices in $\mathscr{B}_{n}^{i}$. Let $B_{n, a, b}$ be the bicyclic graph on $n$ vertices obtained by attaching $a-3$ and $b-3$ pendent vertices to the two vertices of degree 3 of $B_{4}$, respectively, where $a \geq b \geq$ 3 and $a+b=n+2$. Let $B_{n, a, b}^{\prime}$ be the bicyclic graph on $n$ vertices obtained by attaching $a-3$ and $b-3$ pendent vertices to the two vertices of degree 3 of $B_{5}$, respectively, where $a \geq b \geq 3$ and $a+b=n+1$. Then $B_{4} \cong B_{4,3,3}$ and $B_{5} \cong$ $B_{5,3,3}^{\prime}$. See Figure 3 and Figure 4 for an illustration. We first determine the minimum harmonic index for graphs in $\mathscr{B}_{n}$ with matching number 2. 


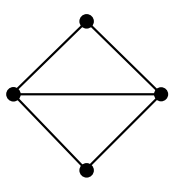

$B_{4}$

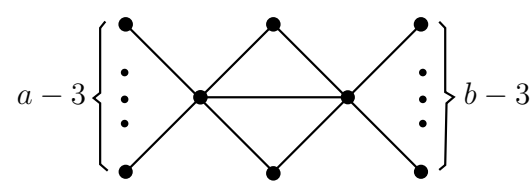

$B_{n, a, b}$

FIGURE 3. The graphs $B_{4}$ and $B_{n, a, b}$.

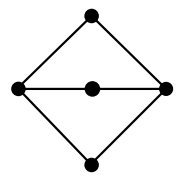

$B_{5}$

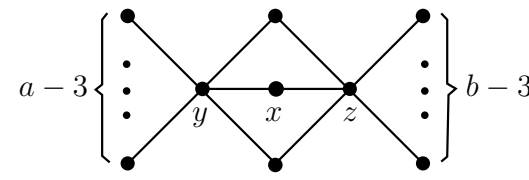

$B_{n, a, b}^{\prime}$

FIGURE 4. The graphs $B_{5}$ and $B_{n, a, b}^{\prime}$.

Theorem 3. Let $G \in \mathscr{B}_{n, 2}$ with $n \geq 4$. Then

$$
H(G) \geq \frac{2}{n+2}+\frac{4}{n+1}+\frac{2(n-4)}{n}+\frac{4}{5}
$$

with equality if and only if $G \cong B_{n, n-1,3}$.

Proof. Since $B_{4}$ is the unique bicyclic graph on 4 vertices in $\mathscr{B}_{4,2}$, we see that the result holds for $n=4$. If $n=5$, then $G \in\left\{F_{i} \mid 1 \leq i \leq 3\right\} \cup B_{5} \cup B_{5,4,3}$, where $F_{i}$ $(1 \leq i \leq 3)$ are shown in Figure 5. It is easy to calculate that $H\left(F_{1}\right)=\frac{73}{30}>H\left(B_{5}\right)=$ $\frac{12}{5}>H\left(F_{2}\right)=\frac{7}{3}>H\left(F_{3}\right)=\frac{23}{10}>\frac{226}{105}=H\left(B_{5,4,3}\right)$, and hence the assertion of the theorem holds. So we may assume that $n \geq 6$. We consider three cases according to the structure of $G$.

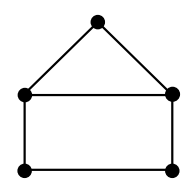

$F_{1}$

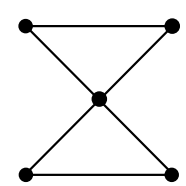

$F_{2}$

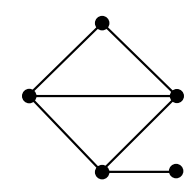

$F_{3}$

FIGURE 5. The graphs $F_{1}, F_{2}$ and $F_{3}$. 
Case 1. $G \cong B_{n, a, b}$, where $a \geq b \geq 3$ and $a+b=n+2$.

Let $f(x)=\frac{4}{x+1}-\frac{8}{x}$. For $x \geq 3$, we have

$$
f^{\prime \prime}(x)=\frac{8}{(x+1)^{3}}-\frac{16}{x^{3}}=\frac{-8\left(x^{3}+6 x^{2}+6 x+2\right)}{x^{3}(x+1)^{3}}<0 .
$$

This implies that $f(x+1)-f(x)$ is decreasing for $x \geq 3$. Suppose $a \geq b \geq 4$. Then

$$
\begin{aligned}
& H\left(B_{n, a+1, b-1}\right)-H\left(B_{n, a, b}\right) \\
&=\left(\frac{4}{(a+1)+2}+\frac{2[(a+1)-3]}{(a+1)+1}+\frac{4}{(b-1)+2}+\frac{2[(b-1)-3]}{(b-1)+1}\right. \\
&\left.+\frac{2}{(a+1)+(b-1)}\right)-\left(\frac{4}{a+2}+\frac{2(a-3)}{a+1}+\frac{4}{b+2}+\frac{2(b-3)}{b+1}+\frac{2}{a+b}\right) \\
&=\left(\frac{4}{a+3}-\frac{12}{a+2}+\frac{8}{a+1}\right)-\left(\frac{4}{b+2}-\frac{12}{b+1}+\frac{8}{b}\right) \\
&= {[f(a+2)-f(a+1)]-[f(b+1)-f(b)]<0, }
\end{aligned}
$$

i.e., $H\left(B_{n, a, b}\right)>H\left(B_{n, a+1, b-1}\right)$ for $a \geq b \geq 4$. So we conclude that $H\left(B_{n, a, b}\right) \geq$ $H\left(B_{n, n-1,3}\right)$ with equality if and only if $a=n-1$ and $b=3$.

Case 2. $G$ is the bicyclic graph obtained by attaching $n-4$ pendent vertices to one vertex of degree 2 of $B_{4}$.

Then

$$
\begin{aligned}
& H(G)-H\left(B_{n, n-1,3}\right) \\
& =\left(\frac{4}{n+1}+\frac{2(n-4)}{n-1}+\frac{4}{5}+\frac{1}{3}\right)-\left(\frac{2}{n+2}+\frac{4}{n+1}+\frac{2(n-4)}{n}+\frac{4}{5}\right) \\
& =\frac{8}{n}-\frac{2}{n+2}-\frac{6}{n-1}+\frac{1}{3}=\left(\frac{2}{n}-\frac{2}{n+2}\right)-\frac{6}{n(n-1)}+\frac{1}{3} \\
& \geq\left(\frac{2}{n}-\frac{2}{n+2}\right)-\frac{6}{6 \cdot(6-1)}+\frac{1}{3}>0 .
\end{aligned}
$$

So Case 2 holds.

Case 3. $G \cong B_{n, a, b}^{\prime}$, where $a \geq b \geq 3$ and $a+b=n+1$.

Let $x$ be one vertex of degree 2 , and let $y, z$ be the two vertices of degree at least 3 in $G$, see Figure 4. Let $G^{\prime}=G-x z+y z$, then $G^{\prime} \cong B_{n, a+1, b}$. By Lemma 3, we have $H(G)>H\left(G^{\prime}\right)$. Hence by the argument in Case 1 , we deduce that $H(G)>$ $H\left(B_{n, n-1,3}\right)$. This completes the proof of the theorem.

The following lemma was proved by Zhu, Liu and Wang [33], which will be used in the following argument.

Lemma 7. Let $G \in \mathscr{B}_{n, m}(n>2 m \geq 6)$ and $G$ contains at least one pendent vertex. Then there exists a maximum matching $M$ and a pendent vertex $u$ in $G$ such that $u$ is not $M$-saturated. 


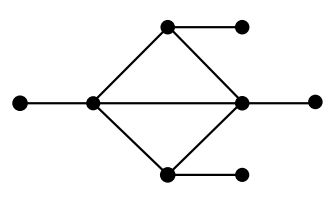

$B_{8}$

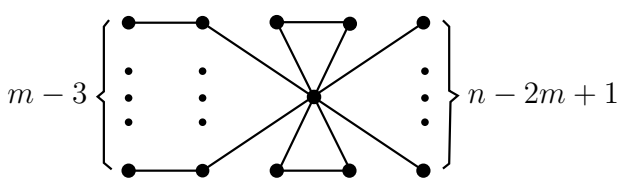

$B_{n, m}$

FIgURE 6. The graphs $B_{8}$ and $B_{n, m}$.

Let $B_{8}$ be the bicyclic graph on 8 vertices obtained by attaching a pendent vertex to every vertex of $B_{4}$. For $3 \leq m \leq\left\lfloor\frac{n}{2}\right\rfloor$, we use $B_{n, m}$ to denote the bicyclic graph on $n$ vertices obtained by attaching $n-2 m+1$ pendent vertices and $m-3$ paths on two vertices to the vertex of degree 4 of $F_{2}$, see Figure 6.

Lemma 8. Let $G \in \mathscr{B}_{2 m, m} \backslash\left\{B_{8}\right\}(m \geq 3)$ and no pendent vertex has neighbor of degree 2. Then

$$
H(G) \geq \frac{2(m+1)}{m+4}+\frac{2}{m+3}+\frac{2(m-3)}{3}+1
$$

with equality if and only if $G \cong B_{6,3}$.

Proof. Let $M$ be a maximum matching in $G$, then $|M|=m$ and every vertex in $G$ is adjacent to at most one pendent vertex. Since $G \in \mathscr{B}_{2 m, m} \backslash\left\{B_{8}\right\}$ and no pendent vertex has neighbor of degree 2 , we see that $G$ can be obtained by attaching some pendent vertices to a bicyclic graph $\tilde{G} \in \tilde{\mathscr{B}}_{k}(m \leq k \leq 2 m)$. We consider two cases according to $G$ contains vertices of degree 2 or not.

Case 1. There is no vertex of degree 2 in $G$.

Then either $k=m$ or $k=m+1$. If $k=m$, then $G$ can be obtained by attaching a pendent vertex to every vertex of a bicyclic graph $\tilde{G} \in \tilde{\mathscr{B}}_{m}$. If $k=m+1$, then $G$ can be obtained by attaching a pendent vertex to every vertex of degree 2 of a bicyclic graph $\tilde{G} \in \mathscr{B}_{m+1}^{1} \cup \mathscr{B}_{m+1}^{4}$.

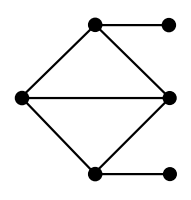

$Q_{1}$

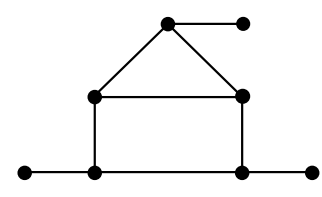

$Q_{2}$

FIGURE 7. The graphs $Q_{1}$ and $Q_{2}$.

If $m=3$, then $\tilde{G} \cong B_{4}$ and $G \cong Q_{1}$ (see Figure 7). Since $H\left(Q_{1}\right)=\frac{8}{3}>\frac{52}{21}=$ $\frac{2 \cdot(3+1)}{3+4}+\frac{2}{3+3}+\frac{2 \cdot(3-3)}{3}+1$, we know that the lemma holds. 
If $m=4$, since we assume $G \not B_{8}$, we have $\tilde{G} \cong F_{1}$ and $G \cong Q_{2}$ (see Figure 7). So the assertion of the lemma holds because $H\left(Q_{2}\right)=\frac{7}{2}>\frac{269}{84}=\frac{2 \cdot(4+1)}{4+4}+\frac{2}{4+3}+$ $\frac{2 \cdot(4-3)}{3}+1$.

Now assume that $m \geq 5$. Then

$$
H(G)= \begin{cases}\frac{5 m}{6}-\frac{59}{420}, & \text { if } \tilde{G} \in \mathscr{B}_{m}^{1} \cup \mathscr{B}_{m}^{4}, \\ \frac{5 m}{6}-\frac{16}{10}, & \text { if } \tilde{G} \in \mathscr{B}_{m}^{2} \cup \mathscr{B}_{m}^{5}, \\ \frac{5 m}{6}-\frac{1}{6}, & \text { if } \tilde{G} \in \mathscr{B}_{m}^{3}, \\ \frac{5 m}{6}+\frac{1}{6}, & \text { if } \tilde{G} \in \mathscr{B}_{m+1}^{1} \cup \mathscr{B}_{m+1}^{4} .\end{cases}
$$

Let $f(x)=\left(\frac{5 x}{6}-\frac{1}{6}\right)-\left(\frac{2(x+1)}{x+4}+\frac{2}{x+3}+\frac{2(x-3)}{3}+1\right)=\frac{x}{6}+\frac{6}{x+4}-\frac{2}{x+3}-\frac{7}{6}$. For $x \geq 5$, we have

$$
f^{\prime}(x)=\frac{1}{6}-\frac{6}{(x+4)^{2}}+\frac{2}{(x+3)^{2}} \geq \frac{1}{6}-\frac{6}{(5+4)^{2}}+\frac{2}{(x+3)^{2}}>0 .
$$

This implies that $f(x)$ is increasing for $x \geq 5$, and thus $f(m) \geq f(5)=\frac{1}{12}>0$, i.e., $H(G)>\frac{2(m+1)}{m+4}+\frac{2}{m+3}+\frac{2(m-3)}{3}+1$.

Case 2. There exists a vertex, say $u$, of degree 2 in $G$.

Let $v$ and $w$ be the two neighbors of $u$ in $G$ such that $d(v)=s \geq 2$ and $d(w)=$ $t \geq 2$. By the symmetry between $v$ and $w$, we may assume that $u v \in M$.

Suppose that no vertex of degree 2 is contained in the cycles of $G$. Since no pendent vertex has neighbor of degree 2 in $G$, we conclude that $\tilde{G} \in \mathscr{B}_{k}^{2}$ and $u$ lies on the path connecting two vertex-disjoint cycles of $G$. Hence $v w \notin E(G)$. Let $G^{\prime}=G-u w+v w$, then $G^{\prime} \in \mathscr{B}_{2 m, m} \backslash\left\{B_{8}\right\}$. By Lemma 3, we have $H(G)>H\left(G^{\prime}\right)$. Comparing with the graph $G$, we see that the number of vertices of degree 2 in $G^{\prime}$ decreases by 1 . Repeating this operation from $G$ to $G^{\prime}$, we finally obtain a bicyclic graph described in Case 1, and hence the result holds.

So we may choose $u$ such that $u$ lies on some cycle of $G$. Let $N(w)=\left\{w_{0}=\right.$ $\left.u, w_{1}, \ldots, w_{t-1}\right\}$, and let $G^{\prime \prime}=G-u w$. Then $G^{\prime \prime}$ is a unicyclic graph on $2 m$ vertices with a perfect matching $M$, i.e., $G^{\prime \prime} \in \mathscr{U}_{2 m, m}$. Note that $2 \leq s, t \leq 5$ and $w$ is adjacent to at most one pendent vertex. Since $\frac{2}{s+2}-\frac{2}{s+1}$ is increasing for $s \geq 2, \frac{2}{t+x}-\frac{2}{t-1+x}$ is increasing for $x \geq 1$ and by Lemma 2(i), we have

$$
\begin{aligned}
H(G)= & H\left(G^{\prime \prime}\right)+\sum_{i=1}^{t-1}\left(\frac{2}{t+d\left(w_{i}\right)}-\frac{2}{t-1+d\left(w_{i}\right)}\right)+\frac{2}{t+2}+\left(\frac{2}{s+2}-\frac{2}{s+1}\right) \\
\geq & H\left(G^{\prime \prime}\right)+\left(\frac{2}{t+1}-\frac{2}{t}\right)+(t-2)\left(\frac{2}{t+2}-\frac{2}{t+1}\right)+\frac{2}{t+2} \\
& +\left(\frac{2}{2+2}-\frac{2}{2+1}\right) \\
= & H\left(G^{\prime \prime}\right)+\left(\frac{2(t-1)}{t+2}-\frac{2(t-3)}{t+1}-\frac{2}{t}\right)-\frac{1}{6}
\end{aligned}
$$




$$
\begin{aligned}
& \geq H\left(G^{\prime \prime}\right)+\left(\frac{2 \cdot(5-1)}{5+2}-\frac{2 \cdot(5-3)}{5+1}-\frac{2}{5}\right)-\frac{1}{6} \\
& =H\left(G^{\prime \prime}\right)-\frac{19}{210}
\end{aligned}
$$

with equalities if and only if $s=2, t=5$, one neighbor of $w$ has degree 1 and the other neighbors of $w$ have degree 2 .

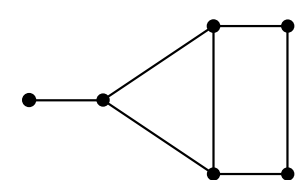

$R_{1}$

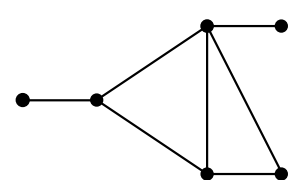

$R_{2}$

FIGURE 8 . The graphs $R_{1}$ and $R_{2}$.

If $G^{\prime \prime} \cong U_{6}$, then either $G^{\prime \prime} \cong R_{1}$ or $G^{\prime \prime} \cong R_{2}$ (see Figure 8). Since $H\left(R_{1}\right)=$ $\frac{14}{5}>H\left(R_{2}\right)=\frac{533}{210}>\frac{52}{21}=\frac{2 \cdot(3+1)}{3+4}+\frac{2}{3+3}+\frac{2 \cdot(3-3)}{3}+1$, the assertion of the lemma holds. If $G^{\prime \prime} \cong U_{8}$, then by $(*)$, we have

$$
\begin{aligned}
H(G) & \geq H\left(U_{8}\right)-\frac{19}{210}=\frac{347}{105}-\frac{19}{210}=\frac{45}{14} \\
& >\frac{269}{84}=\frac{2 \cdot(4+1)}{4+4}+\frac{2}{4+3}+\frac{2 \cdot(4-3)}{3}+1,
\end{aligned}
$$

and the result holds. So suppose that $G^{\prime \prime} \nsupseteq U_{6}, U_{8}$. It follows from Lemma 2(i) that

$$
\begin{aligned}
& \frac{2[(m+2)-1]}{(m+2)+2}-\frac{2[(m+2)-3]}{(m+2)+1}-\frac{2}{m+2} \\
& \leq \frac{2 \cdot[(3+2)-1]}{(3+2)+2}-\frac{2 \cdot[(3+2)-3]}{(3+2)+1}-\frac{2}{3+2}=\frac{8}{105}
\end{aligned}
$$

since $m \geq 3$. Then by $(*)$ and Theorem 1 , we have

$$
\begin{aligned}
H(G) & \geq H\left(G^{\prime \prime}\right)-\frac{19}{210} \\
& \geq\left(\frac{2 m}{m+3}+\frac{2}{m+2}+\frac{2(m-2)}{3}+\frac{1}{2}\right)-\frac{19}{210} \\
& =\left(\frac{2 m}{m+3}+\frac{2}{m+2}+\frac{2(m-3)}{3}+1\right)+\frac{8}{105} \\
& \geq\left(\frac{2 m}{m+3}+\frac{2}{m+2}+\frac{2(m-3)}{3}+1\right)
\end{aligned}
$$




$$
\begin{aligned}
& +\left(\frac{2[(m+2)-1]}{(m+2)+2}-\frac{2[(m+2)-3]}{(m+2)+1}-\frac{2}{m+2}\right) \\
= & \frac{2(m+1)}{m+4}+\frac{2}{m+3}+\frac{2(m-3)}{3}+1
\end{aligned}
$$

with equalities if and only if $s=2, t=5, G^{\prime \prime} \cong U_{2 m, m}$ and $m=3$, i.e., $G \cong B_{6,3}$. This finishes the proof of the lemma.

Theorem 4. Let $G \in \mathscr{B}_{2 m, m} \backslash\left\{B_{8}\right\}$, where $m \geq 3$. Then

$$
H(G) \geq \frac{2(m+1)}{m+4}+\frac{2}{m+3}+\frac{2(m-3)}{3}+1
$$

with equality if and only if $G \cong B_{2 m, m}$.

Proof. We prove Theorem 4 by induction on $m$. If $m=3$, then by Lemma 7, we may assume that there exists a pendent vertex in $G$ whose neighbor is a vertex of degree 2 . Hence $G$ is the bicyclic graph obtained from $B_{4}$ by attaching a path on two vertices to either one vertex of degree 3 or one vertex of degree 2 . Then we have $H(G) \geq \frac{289}{105}>\frac{52}{21}=H\left(B_{6,3}\right)$, and the assertion of the theorem holds. So we may assume that $m \geq 4$ and the result holds for graphs in $\mathscr{B}_{2(m-1), m-1} \backslash\left\{B_{8}\right\}$. Let $M$ be a maximum matching in $G$, then $|M|=m$. If no pendent vertex has neighbor of degree 2 in $G$, then by Lemma 7, we see that the result holds.

Now suppose that there exists a pendent vertex $u$ in $G$ whose neighbor $v$ is a vertex of degree 2. Let $w$ be the neighbor of $v$ different from $u$ with $d(w)=t \geq 2$, and let $G^{\prime}=G-u-v$. Then $u v \in M$ and $G^{\prime} \in \mathscr{B}_{2(m-1), m-1}$. Since $M$ contains exactly one edge incident with $w$ and there are $m+1$ edges of $G$ outside $M$, we have $t \leq m+2$. Note that $w$ is adjacent to at most one pendent vertex in $G$.

If $G^{\prime} \cong B_{8}$, then $t \leq 5$. By Lemma 1(i) and Lemma 2(i), we have

$$
\begin{aligned}
H(G) & \geq H\left(B_{8}\right)+\frac{2(t-1)}{t+2}-\frac{2(t-3)}{t+1}-\frac{2}{t}+\frac{2}{3} \\
& \geq \frac{447}{140}+\frac{2 \cdot(5-1)}{5+2}-\frac{2 \cdot(5-3)}{5+1}-\frac{2}{5}+\frac{2}{3}=\frac{551}{140}>\frac{47}{12}=H\left(U_{10,5}\right),
\end{aligned}
$$

and hence the assertion of the theorem holds.

So we may further assume that $G^{\prime} \not B_{8}$. Then by Lemma 1(i), Lemma 2(i) and the induction hypothesis, we conclude that

$$
\begin{aligned}
H(G) \geq & H\left(G^{\prime}\right)+\frac{2(t-1)}{t+2}-\frac{2(t-3)}{t+1}-\frac{2}{t}+\frac{2}{3} \\
\geq & \left(\frac{2[(m-1)+1]}{(m-1)+4}+\frac{2}{(m-1)+3}+\frac{2[(m-1)-3]}{3}+1\right) \\
& +\frac{2[(m+2)-1]}{(m+2)+2}-\frac{2[(m+2)-3]}{(m+2)+1}-\frac{2}{m+2}+\frac{2}{3}
\end{aligned}
$$




$$
=\frac{2(m+1)}{m+4}+\frac{2}{m+3}+\frac{2(m-3)}{3}+1
$$

with equalities if and only if $G^{\prime} \cong B_{2(m-1), m-1}$ and $t=m+2$, i.e., $G \cong B_{2 m, m}$. So Theorem 4 holds.

Since $H\left(B_{8,4}\right)=\frac{269}{84}>\frac{447}{140}=H\left(B_{8}\right)$, by Theorem 4, we immediately obtain the following result.

Corollary 4. Let $G \in \mathscr{B}_{8,4}$, then $H(G) \geq \frac{447}{140}$ with equality if and only if $G \cong B_{8}$.

We now present the minimum harmonic index for graphs in $\mathscr{B}_{n, m} \backslash\left\{B_{8}\right\}$, where $3 \leq m \leq\left\lfloor\frac{n}{2}\right\rfloor$.

Theorem 5. Let $G \in \mathscr{B}_{n, m} \backslash\left\{B_{8}\right\}$, where $3 \leq m \leq\left\lfloor\frac{n}{2}\right\rfloor$. Then

$$
H(G) \geq \frac{2(m+1)}{n-m+4}+\frac{2(n-2 m+1)}{n-m+3}+\frac{2(m-3)}{3}+1
$$

with equality if and only if $G \cong B_{n, m}$.

Proof. We prove the theorem by induction on $n$. If $n=2 m$, then by Theorem 4, the assertion of the theorem holds. So we may assume that $n>2 m$ and the result holds for graphs in $\mathscr{B}_{n-1, m} \backslash\left\{B_{8}\right\}$. If there is no pendent vertex in $G$, then $G \in \tilde{\mathscr{B}}_{n}$ and $n=2 m+1$. It is easy to check that

$$
H(G)= \begin{cases}m+\frac{13}{30}, & \text { if } G \in \mathscr{B}_{2 m+1}^{1} \cup \mathscr{B}_{2 m+1}^{4}, \\ m+\frac{2}{5}, & \text { if } G \in \mathscr{B}_{2 m+1}^{2} \cup \mathscr{B}_{2 m+1}^{5}, \\ m+\frac{1}{3}, & \text { if } G \in \mathscr{B}_{2 m+1}^{3}\end{cases}
$$

This implies that

$$
\begin{aligned}
& H(G)-H\left(B_{2 m+1, m)}\right. \\
& \geq\left(m+\frac{1}{3}\right)-\left(\frac{2(m+1)}{(2 m+1)-m+4}+\frac{2[(2 m+1)-2 m+1]}{(2 m+1)-m+3}+\frac{2(m-3)}{3}+1\right) \\
& =\frac{m}{3}+\frac{8}{m+5}-\frac{4}{m+4}-\frac{2}{3}=\frac{m-2}{3}+\frac{4(m+3)}{(m+4)(m+5)}>0,
\end{aligned}
$$

i.e., $H(G)>H\left(B_{2 m+1, m}\right)$.

So we may assume that $G$ contains at least one pendent vertex. Then by Lemma 7, there exists a maximum matching $M$ and a pendent vertex $u$ in $G$ such that $u$ is not $M$-saturated. Let $v$ be the unique neighbor of $u$ with $d(v)=s \geq 2$, and let $G^{\prime}=G-u$. Then $G^{\prime} \in \mathscr{B}_{n-1, m}$. Since $M$ contains exactly one edge incident with $v$ and there are $n+1-m$ edges of $G$ outside $M$, we have $s \leq n-m+2$. Let $r$ be the number of pendant neighbors of $v$ in $G$, where $1 \leq r \leq s-1$. Note that at least $r-1$ pendant neighbors of $v$ are not $M$-saturated, and there are $n-2 m$ vertices are not $M$-saturated in $G$. Then $r \leq n-2 m+1$. 
If $G^{\prime} \cong B_{8}$, then $n=9, m=4$ and $s \leq 5$. By Lemma 1(ii) (with $k=n-2 m+1=$ 2) and Lemma 2(ii), we deduce that

$$
\begin{aligned}
H(G) & \geq H\left(B_{8}\right)+\frac{2(s-2)}{s+2}+\frac{2(4-s)}{s+1}-\frac{2}{s} \\
& \geq \frac{447}{140}+\frac{2 \cdot(5-2)}{5+2}+\frac{2 \cdot(4-5)}{5+1}-\frac{2}{5}=\frac{1393}{420}>\frac{59}{18}=H\left(B_{9,4}\right),
\end{aligned}
$$

and hence the assertion of the theorem holds.

Therefore we may assume that $G^{\prime} \nRightarrow B_{8}$. Then by Lemma 1(ii) (with $k=n-$ $2 m+1$ ), Lemma 2(ii) and the induction hypothesis, we have

$$
\begin{aligned}
H(G) \geq & H\left(G^{\prime}\right)+\frac{2[s-(n-2 m+1)]}{s+2}+\frac{2[2(n-2 m+1)-s]}{s+1} \\
& -\frac{2[(n-2 m+1)-1]}{s} \\
\geq & \left(\frac{2(m+1)}{(n-1)-m+4}+\frac{2[(n-1)-2 m+1]}{(n-1)-m+3}+\frac{2(m-3)}{3}+1\right) \\
& +\frac{2[(n-m+2)-(n-2 m+1)]}{(n-m+2)+2}+\frac{2[2(n-2 m+1)-(n-m+2)]}{(n-m+2)+1} \\
& -\frac{2[(n-2 m+1)-1]}{n-m+2} \\
= & \frac{2(m+1)}{n-m+4}+\frac{2(n-2 m+1)}{n-m+3}+\frac{2(m-3)}{3}+1
\end{aligned}
$$

with equalities if and only if $G^{\prime} \cong B_{n-1, m}, s=n-m+2$ and $r=n-2 m+1$, i.e., $G \cong B_{n, m}$. This completes the proof of the theorem.

We can also determine the minimum harmonic index for graphs in $\mathscr{B}_{n}$ (see also in [31]) by using Theorem 3 and Theorem 5 .

Corollary 5. Let $G \in \mathscr{B}_{n}$ with $n \geq 4$. Then

$$
H(G) \geq \frac{2}{n+2}+\frac{4}{n+1}+\frac{2(n-4)}{n}+\frac{4}{5}
$$

with equality if and only if $G \cong B_{n, n-1,3}$.

Proof. Let $M$ be a maximum matching in $G$, then $2 \leq|M|=m \leq\left\lfloor\frac{n}{2}\right\rfloor$ (since $n \geq 4$ ). If $m=2$, then the result follows immediately from Theorem 3 .

If $m=3$, then by Theorem 5 , we have

$$
\begin{aligned}
H(G) & \geq \frac{2 \cdot(3+1)}{n-3+4}+\frac{2(n-2 \cdot 3+1)}{n-3+3}+\frac{2 \cdot(3-3)}{3}+1 \\
& =\frac{8}{n+1}+\frac{2(n-5)}{n}+1
\end{aligned}
$$


with equality if and only if $G \cong B_{n, 3}$. Note that in this case $n \geq 6$. Since

$$
\begin{aligned}
& H\left(B_{n, 3}\right)-H\left(B_{n, n-1,3}\right) \\
& =\left(\frac{8}{n+1}+\frac{2(n-5)}{n}+1\right)-\left(\frac{2}{n+2}+\frac{4}{n+1}+\frac{2(n-4)}{n}+\frac{4}{5}\right) \\
& =\left(\frac{4}{n+1}-\frac{2}{n+2}-\frac{2}{n}\right)+\frac{1}{5}=\frac{-4}{n(n+1)(n+2)}+\frac{1}{5} \\
& \geq \frac{-4}{6 \cdot(6+1) \cdot(6+2)}+\frac{1}{5}=\frac{79}{420}>0,
\end{aligned}
$$

we know that the assertion of the corollary holds.

So we may assume that $m \geq 4$. If $G \cong B_{8}$, then $H(G)=\frac{447}{140}>\frac{22}{9}=H\left(B_{8,7,3}\right)$, we see that Corollary 5 holds. Now suppose that $G \not B_{8}$. Then by Theorem 5 and Lemma 3, we see that $H(G) \geq H\left(B_{n, m}\right)>H\left(B_{n, m-1}\right)>\cdots>H\left(B_{n, 3}\right)>$ $H\left(B_{n, n-1,3}\right)$. This finishes the proof of the corollary.

\section{REFERENCES}

[1] A. Chang and F. Tian, "On the spectral radius of unicyclic graphs with perfect matching," Linear Algebra Appl., vol. 370, pp. 237-250, 2003.

[2] H. Deng, S. Balachandran, S. K. Ayyaswamy, and V. B. Venkatakrishnan, "On the harmonic index and the chromatic number of a graph," Discrete Appl. Math., vol. 161, no. 16-17, pp. 2740-2744, 2013.

[3] H. Deng, S. Balachandran, S. K. Ayyaswamy, and V. B. Venkatakrishnan, "On harmonic indices of trees, unicyclic graphs and bicyclic graphs," Ars Combin., in press.

[4] Z. Du and B. Zhou, "On sum-connectivity index of bicyclic graphs," Bull. Malays. Math. Sci. Soc., vol. 35, no. 1, pp. 101-117, 2012.

[5] Z. Du, B. Zhou, and N. Trinajstić, "Minimum sum-connectivity indices of trees and unicyclic graphs of a given matching number," J. Math. Chem., vol. 47, no. 2, pp. 842-855, 2010.

[6] S. Fajtlowicz, "On conjectures of Graffiti-II," Congr. Numer., vol. 60, pp. 187-197, 1987.

[7] O. Favaron, M. Mahéo, and J. F. Saclé, "Some eigenvalue properties in graphs (conjectures of Graffiti-II)," Discrete Math., vol. 111, no. 1-3, pp. 197-220, 1993.

[8] B. Furtula, I. Gutman, and M. Dehmer, "On structure-sensitivity of degree-based topological indices," Appl. Math. Comput., vol. 219, no. 17, pp. 8973-8978, 2013.

[9] I. Gutman and B. Furtula, Recent Results in the Theory of Randic Index. Kragujevac: University of Kragujevac, 2008.

[10] I. Gutman, B. Furtula, and C. Elphick, "Three new/old vertex-degree-based topological indices," MATCH Commun. Math. Comput. Chem., vol. 72, no. 3, pp. 617-632, 2014.

[11] I. Gutman and J. Tošović, "Testing the quality of molecular structure descriptors. Vertex-degreebased topological indices," J. Serb. Chem. Soc., vol. 78, no. 6, pp. 805-810, 2013.

[12] A. Ilić, "Note on the harmonic index of a graph," preprint.

[13] J. Li and B. Zhou, "On the modified Randić index of trees, unicyclic graphs and bicyclic graphs," Miskolc Math. Notes, vol. 13, no. 2, pp. 415-427, 2012.

[14] X. Li and I. Gutman, Mathematical Aspects of Randić-Type Molecular Structure Descriptors. Kragujevac: University of Kragujevac, 2006.

[15] X. Li and Y. Shi, "A survey on the Randić index," MATCH Commun. Math. Comput. Chem., vol. 59, no. 1, pp. 127-156, 2008. 
[16] H. Liu, X. Pan, and J. Xu, "On the Randić index of unicyclic conjugated molecules," J. Math. Chem., vol. 40, no. 2, pp. 135-143, 2006.

[17] J. Liu, "On harmonic index and diameter of graphs," J. Appl. Math. Phys., vol. 1, no. 3, pp. 5-6, 2013.

[18] J. Liu, "On the harmonic index of triangle-free graphs," Appl. Math., vol. 4, no. 8, pp. 1204-1206, 2013.

[19] J. Rada and R. Cruz, "Vertex-degree-based topological indices over graphs," MATCH Commun. Math. Comput. Chem., vol. 72, no. 3, pp. 603-616, 2014.

[20] M. Randić, "On characterization of molecular branching," J. Am. Chem. Soc., vol. 97, no. 23, pp. 6609-6615, 1975.

[21] I. Tomescu and M. K. Jamil, "Maximum general sum-connectivity index for trees with given independence number," MATCH Commun. Math. Comput. Chem., vol. 72, no. 3, pp. 715-722, 2014.

[22] I. Tomescu and S. Kanwal, "Ordering trees having small general sum-connectivity index," MATCH Commun. Math. Comput. Chem., vol. 69, no. 3, pp. 535-548, 2013.

[23] R. Wu, Z. Tang, and H. Deng, "A lower bound for the harmonic index of a graph with minimum degree at least two," Filomat, vol. 27, no. 1, pp. 51-55, 2013.

[24] R. Wu, Z. Tang, and H. Deng, "On the harmonic index and the girth of a graph," Utilitas Math., vol. 91, pp. 65-69, 2013.

[25] X. Xu, "Relationships between harmonic index and other topological indices," Appl. Math. Sci., vol. 6, no. 41, pp. 2013-2018, 2012.

[26] L. Yang and H. Hua, "The harmonic index of general graphs, nanocones and triangular benzenoid graphs," Optoelectron. Adv. Mater. - Rapid Commun., vol. 6, no. 5-6, pp. 660-663, 2012.

[27] A. Yu and F. Tian, "On the spectral radius of unicyclic graphs," MATCH Commum. Math. Comput. Chem., vol. 51, no. 1, pp. 97-109, 2004.

[28] L. Zhong, "The harmonic index for graphs," Appl. Math. Lett., vol. 25, no. 3, pp. 561-566, 2012.

[29] L. Zhong, "The harmonic index on unicyclic graphs," Ars Combin., vol. 104, pp. 261-269, 2012.

[30] L. Zhong and K. Xu, "The harmonic index for bicyclic graphs," Utilitas Math., vol. 90, pp. 23-32, 2013.

[31] L. Zhong and K. Xu, "Inequalities between vertex-degree-based topological indices," MATCH Commun. Math. Comput. Chem., vol. 71, no. 3, pp. 627-642, 2014.

[32] B. Zhou and N. Trinajstić, "On general sum-connectivity index," J. Math. Chem., vol. 47, no. 1, pp. 210-218, 2010.

[33] Y. Zhu, G. Liu, and J. Wang, "On the Randić index of bicyclic conjugated molecules," in Recent Results in the Theory of Randic Index, I. Gutman and B. Furtula, Eds. Kragujevac: University of Kragujeva, 2008, pp. 133-144.

Author's address

Lingping Zhong

Nanjing University of Aeronautics and Astronautics, Department of Mathematics, Nanjing 210016, P. R. China

E-mail address: zhong@nuaa.edu.cn 